\title{
On Parallel Curves Obtained by a Space Curve in Minkowski Space
}

\author{
Muhammed T. Sariaydin ${ }^{1 *}$, Talat Körpinar ${ }^{2}$ \\ ${ }^{1}$ Selçuk University, Department of Mathematics, Konya, Turkey. \\ 2 Mus Alparslan University, Department of Mathematics, Mus, Turkey. \\ * Corresponding author. Tel+90 50778923 17; email: talatsariaydin@gmail.com \\ Manuscript submitted September 13, 2018; accepted November 8, 2018. \\ doi: 10.17706/ijapm.2019.9.1.38-46
}

\begin{abstract}
This paper presents parallel curves according to modified frame in Minkowski 3-space. Firstly, it is investigated parallel curves of a given curve via the modified frame with curvature in $\mathrm{E}_{1}^{3}$. We also give parallel curves of a given curve via the modified frame with torsion in $\mathrm{E}_{1}^{3}$.
\end{abstract}

Key words: Adjoint curve, parallel curve, Minkowski space.

\section{Introduction}

The Frenet-Serret frame which has an important place in Euclidean space is obtained continuously differentiable non-degenerate curves. For this reason, the studies related to the Frenet frame are often used in differential geometry. One of the most important of them is Karacan's study. Karacan described new frames, called modified orthogonal frame, with the help of the frenet frame in 2017. These frames have created both curvature and torsion of a space curve in $\mathrm{E}^{3}$. Karacan also described these frames in Minkowski 3-space. Because these frames are newly defined, there is no study in the literature, [1], and [2].

In the literature, special curves are often used in Euclidean space and Minkowski space. For example, Bukucu investigates the spherical curves with Modified Orthogonal Frame, Körpınar gives some theorems related to tangent Bishop spherical images of a biharmonic b-slant helix, and Yllmaz study spherical images according to bishop frame, [2]-[4].

The rest of this paper is organized as follows. In Section 2, we give properties and the basic concepts of curves in Minkowski 3-space. In Section 3, we give the parallel curves of a space curve according to the modified orthogonal frame with curvature and torsion in Minkowski 3-space. Finally, we give a new theorem defined the adjoint curve of parallel curves of timelike space curves in Minkowski 3-space.

\section{Preliminaries}

Let us consider Minkowski 3-space $\mathrm{E}_{1}^{3}=\left(\mathrm{E}_{1}^{3},(-,+,+)\right)$ and let the Lorentzian inner product of $X=\left(x_{1}, x_{2}, x_{3}\right)$ and $Y=\left(y_{1}, y_{2}, y_{3}\right)$ be

$$
\langle X, Y\rangle=-x_{1} y_{1}+x_{2} y_{2}+x_{3} y_{3}
$$

A vector $\mathrm{X} \in \mathrm{E}_{1}^{3}$ is called a spacelike vector when $\langle X, X\rangle>0$ or $X=0$. It is called timelike and null 
vector in case of $\langle X, X\rangle<0$ and $\langle X, X\rangle=0$ for $X \neq 0$, respectively.

The vector product of vectors $X=\left(x_{1}, x_{2}, x_{3}\right)$ and $Y=\left(y_{1}, y_{2}, y_{3}\right)$ in $\mathrm{E}_{1}^{3}$ is defined by, [5],

$$
X \times Y=\left(x_{2} y_{3}-x_{3} y_{2}, x_{1} y_{3}-x_{3} y_{1}, x_{2} y_{1}-x_{1} y_{2}\right)
$$

A surface in $E_{1}^{3}$ is called a timelike surface if the normal vector field on the surface is a spacelike vector and is called spacelike surface if the normal vector field on the surface is a timelike vector, [6].

Theorem 2.1 Assume that $\{t, n, b\}$ is the frenet frame of unit speed curve in $\mathrm{E}_{1}^{3}$. If $\{T, N, B\}$ is the modified orthogonal frame with curvature and $T=t, N=\kappa n, B=\kappa b$, then

$$
\left[\begin{array}{c}
T^{\prime} \\
N^{\prime} \\
B^{\prime}
\end{array}\right]=\left(\begin{array}{ccc}
0 & 1 & 0 \\
\kappa^{2} & \frac{\kappa^{\prime}}{\kappa} & \tau \\
0 & -\tau & \frac{\kappa^{\prime}}{\kappa}
\end{array}\right)\left[\begin{array}{c}
T \\
N \\
B
\end{array}\right],
$$

where $\alpha$ is timelike curve.

$$
\left[\begin{array}{c}
T^{\prime} \\
N^{\prime} \\
B^{\prime}
\end{array}\right]=\left(\begin{array}{ccc}
0 & 1 & 0 \\
-\kappa^{2} & \frac{\kappa^{\prime}}{\kappa} & \tau \\
0 & \tau & \frac{\kappa^{\prime}}{\kappa}
\end{array}\right)\left[\begin{array}{l}
T \\
N \\
B
\end{array}\right],
$$

where $\alpha$ is spacelike curve with a spacelike principal normal.

$$
\left[\begin{array}{c}
T^{\prime} \\
N^{\prime} \\
B^{\prime}
\end{array}\right]=\left(\begin{array}{ccc}
0 & 1 & 0 \\
\kappa^{2} & \frac{\kappa^{\prime}}{\kappa} & \tau \\
0 & \tau & \frac{\kappa^{\prime}}{\kappa}
\end{array}\right)\left[\begin{array}{c}
T \\
N \\
B
\end{array}\right],
$$

where $\alpha$ is spacelike curve with a spacelike binormal, [1].

Lemma 2.2 Two curves $\alpha, \beta: I \rightarrow \mathrm{E}^{3}$ are parallel if their velocity vectors $\alpha^{\prime}(s)$ and $\beta^{\prime}(s)$ are parallel for each $s$. In this case, if $\alpha\left(s_{0}\right)=\beta\left(s_{0}\right)$ for some $s_{0}$ in $I$, then $\alpha=\beta$ [7].

Theorem 2.3 If $\alpha, \beta: I \rightarrow \mathrm{E}^{3}$ are unit-speed curves such that $\kappa_{\alpha}=\kappa_{\beta}$ and $\tau_{\alpha}=\tau_{\beta}$, then $\alpha$ and $\beta$ are congruent, [7]. 
Theorem 2.4 Assume that $\alpha$ is unit speed curve in $\mathrm{E}^{3}$ and $\{T, N, B\}$ is the Frenet frame of the space curve $\alpha$, [8]. Then, the adjoint curve of $\alpha$ is

$$
\beta(s)=\int_{s_{0}}^{s} B(s) d s
$$

\section{Parallel Curves in 3-Dimensional Minkowski Space}

Let $\alpha: I \rightarrow \mathrm{E}^{3}$ be a regular curve with parametrized by arc-length. We obtain

$$
\begin{gathered}
(\alpha(s)-P)^{2}=t^{2}, \\
\alpha^{\prime}(s)(\alpha(s)-P)=0, \\
\alpha^{\prime \prime}(s)(\alpha(s)-P)+\left(\alpha^{\prime}(s)\right)^{2}=0,
\end{gathered}
$$

for the point $P,[1]$.

Theorem 3.1 Assume that $\alpha: I \rightarrow \mathrm{E}_{1}^{3}$ is timelike curve with parametrized by arc-length in $\mathrm{E}_{1}^{3}$. If $\{T, N, B\}$ is the modified orthogonal frame with curvature, then a parallel curve of $\alpha$ is

$$
P_{ \pm}=\alpha+\frac{1}{\kappa^{2}} N \pm \frac{\sqrt{t^{2} \kappa^{2}-1}}{\kappa^{2}} B
$$

Proof. Suppose that the above equations are provided. Then, we can write

$$
\alpha-P=\mu_{1} N+\eta_{1} B
$$

where $\mu_{1}$ and $\eta_{1}$ are appropriate coefficients. Because $\alpha$ is a timelike curve, the following equations can be written as

$$
\left(\alpha^{\prime}\right)^{2}=T^{2}=-1 \text { and } \alpha^{\prime \prime}=T^{\prime}=N
$$

Then, we can write easily

$$
N\left(\mu_{1} N+\eta_{1} B\right)-1=0 .
$$

From (5),

$$
\mu_{1}=\frac{1}{\kappa^{2}}
$$


Also, if we write at (6) instead of the left side of $(1)$, then we get

$$
\eta_{1}= \pm \frac{\sqrt{t^{2} \kappa^{2}-1}}{\kappa^{2}}
$$

Considering together (6) and (7) equations, we get

$$
P_{ \pm}=\alpha+\frac{1}{\kappa^{2}} N \pm \frac{\sqrt{t^{2} \kappa^{2}-1}}{\kappa^{2}} B
$$

Theorem 3.2 Assume that $\alpha: I \rightarrow E_{1}^{3}$ is spacelike curve with timelike principal normal in $\mathrm{E}_{1}^{3}$. If $\{T, N, B\}$ is the modified orthogonal frame with curvature, then a parallel curve of $\alpha$ is

$$
P_{ \pm}=\alpha+\frac{1}{\kappa^{2}} N \pm \frac{\sqrt{t^{2} \kappa^{2}+1}}{\kappa^{2}} B .
$$

Theorem 3.3 Assume that $\alpha: I \rightarrow E_{1}^{3}$ is spacelike curve with timelike binormal in $\mathrm{E}_{1}^{3}$. If $\{T, N, B\}$ is the modified orthogonal frame with curvature, then a parallel curve of $\alpha$ is

$$
P_{ \pm}=\alpha-\frac{1}{\kappa^{2}} N \pm \frac{\sqrt{1-t^{2} \kappa^{2}}}{\kappa^{2}} B
$$

Theorem 3.4 Assume that $\alpha: I \rightarrow E_{1}^{3}$ is timelike curve in $\mathrm{E}_{1}^{3}$. If $\{T, N, B\}$ is the modified orthogonal frame with torsion, then a parallel curve of $\alpha$ is

$$
P_{ \pm}=\alpha+\frac{1}{\kappa \tau} N \pm \frac{\sqrt{t^{2} \kappa^{2}-1}}{\kappa \tau} B
$$

Proof. Suppose that the above equations are provided. Then, we can write

$$
\alpha-P=\mu_{2} N+\eta_{2} B
$$

where $\mu_{2}$ and $\eta_{2}$ are appropriate coefficients. Because $\alpha$ is a timelike curve, the following equations can be written as

$$
\left(\alpha^{\prime}\right)^{2}=T^{2}=-1 \quad \text { and } \quad \alpha^{\prime \prime}=T^{\prime}=\frac{\kappa}{\tau} N
$$

Then, we can write easily

$$
\frac{\kappa}{\tau} N\left(\mu_{2} N+\eta_{2} B\right)-1=0
$$


From (10),

$$
\mu_{2}=\frac{1}{\kappa \tau}
$$

After simple computation, we get

$$
\eta_{2}= \pm \frac{\sqrt{t^{2} \kappa^{2}-1}}{\kappa \tau}
$$

Considering together (11) and (12) equations, we get

$$
P_{ \pm}=\alpha+\frac{1}{\kappa \tau} N \pm \frac{\sqrt{t^{2} \kappa^{2}-1}}{\kappa \tau} B
$$

Theorem 3.5 Assume that $\alpha: I \rightarrow E_{1}^{3}$ is spacelike curve with timelike principal normal in $\mathrm{E}_{1}^{3}$. If $\{T, N, B\}$ is the modified orthogonal frame with torsion, then a parallel curve of $\alpha$ is

$$
P_{ \pm}=\alpha+\frac{1}{\kappa \tau} N \pm \frac{\sqrt{t^{2} \kappa^{2}+1}}{\kappa \tau} B
$$

Theorem 3.6 Assume that $\alpha: I \rightarrow E_{1}^{3}$ is spacelike curve with timelike binormal in $\mathrm{E}_{1}^{3}$. If $\{T, N, B\}$ is the modified orthogonal frame with torsion, then a parallel curve of $\alpha$ is

$$
P_{ \pm}=\alpha-\frac{1}{\kappa \tau} N \pm \frac{\sqrt{1-t^{2} \kappa^{2}}}{\kappa \tau} B
$$

Example 3.7 Let us consider a unit speed curve in $\mathrm{E}_{1}{ }^{3}$ by

$$
\alpha=\alpha(s)\left(a \cosh \frac{s}{c}, a \sinh \frac{s}{c}, \frac{b s}{c}\right) .
$$

Then, frenet frame of spacelike curve $\alpha$ in $\mathrm{E}_{1}^{3}$ is

$$
\begin{aligned}
t(s) & =\left(\frac{3}{5} \sinh \frac{s}{5}, \frac{3}{5} \cosh \frac{s}{5}, \frac{4}{5}\right), \\
n(s) & =\left(\cosh \frac{s}{5}, \sinh \frac{s}{5}, 0\right), \\
b(s) & =\left(\frac{4}{5} \sinh \frac{s}{5}, \frac{4}{5} \cosh \frac{s}{5}, \frac{3}{5}\right), \\
\kappa(s) & = \\
\tau(s) & =\frac{3}{25},
\end{aligned}
$$


where $a=3, b=4, c=5$. Also, the modified orthogonal frame with curvature and torsion of spacelike curve $\alpha$, respectively, are written as

$$
\begin{aligned}
& T_{\kappa}(s)=\left(\frac{3}{5} \sinh \frac{s}{5}, \frac{3}{5} \cosh \frac{s}{5}, \frac{4}{5}\right), \\
& N_{\kappa}(s)=\left(\frac{3}{25} \cosh \frac{s}{5}, \frac{3}{25} \sinh \frac{s}{5}, 0\right), \\
& B_{\kappa}(s)=\left(\frac{12}{125} \sinh \frac{s}{5}, \frac{12}{125} \cosh \frac{s}{5},-\frac{9}{125}\right),
\end{aligned}
$$

And

$$
\begin{aligned}
& T_{\tau}(s)=\left(\frac{3}{5} \sinh \frac{s}{5}, \frac{3}{5} \cosh \frac{s}{5}, \frac{4}{5}\right), \\
& N_{\tau}(s)=\left(\frac{4}{25} \cosh \frac{s}{5}, \frac{4}{25} \sinh \frac{s}{5}, 0\right), \\
& B_{\tau}(s)=\left(\frac{16}{125} \sinh \frac{s}{5}, \frac{16}{125} \cosh \frac{s}{5},-\frac{12}{125}\right) .
\end{aligned}
$$

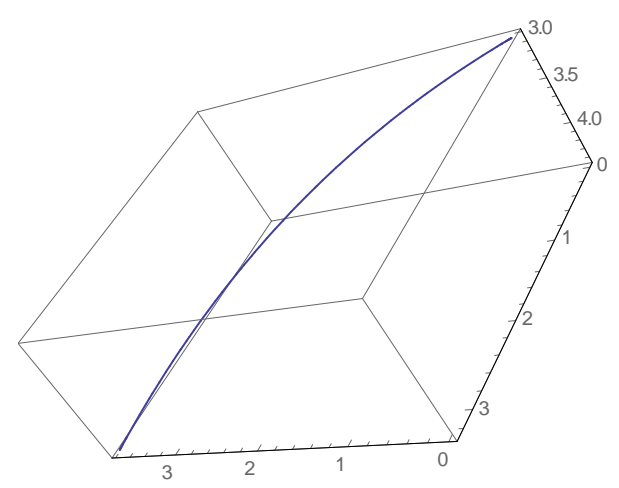

Fig. 1. The curve $\alpha$.

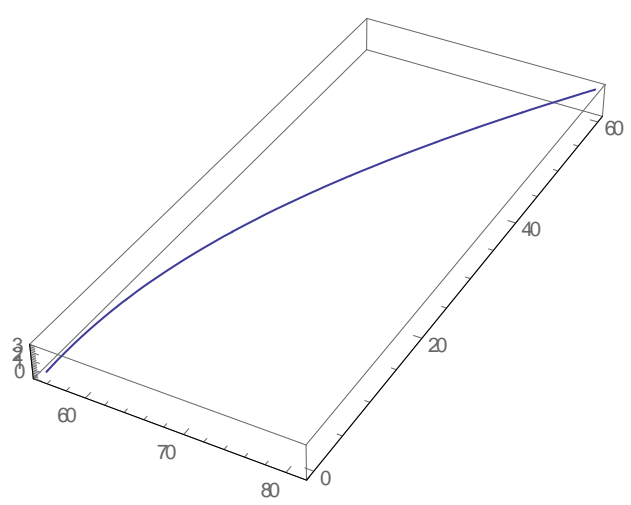

Fig. 2. Parallel curve of the Curve $\alpha$.

Theorem 3.8 Let $\alpha: I \rightarrow E_{1}^{3}$ be timelike curve with parametrized by arc-length in $\mathrm{E}_{1}^{3}$ and 
$P=\alpha+\sigma_{1} N+\sigma_{2} B$, where $\sigma_{1}$ is $\frac{1}{\kappa^{2}}, \sigma_{2}$ is $\frac{\sqrt{t^{2} \kappa^{2}-1}}{\kappa^{2}}$, be a parallel curve of $\alpha$. Then, the frenet vector field of the parallel curve are

$$
\begin{aligned}
& t=\left(1+\kappa^{2} \sigma_{1}\right) T+\left(\sigma_{1}^{\prime}+\sigma_{1} \frac{\kappa^{\prime}}{\kappa}-\sigma_{2} \tau\right) N+\left(\sigma_{1} \tau+\sigma_{2}^{\prime}+\sigma_{2} \frac{\kappa^{\prime}}{\kappa}\right) B \\
& n=\frac{1}{\pi}\left(\left(\mu_{1}^{\prime}+\mu_{2}^{\prime} \kappa^{2}\right) T+\left(\mu_{1}+\mu_{2}^{\prime}+\mu_{2} \frac{\kappa^{\prime}}{\kappa}-\tau \mu_{3}\right) N+\left(\mu_{2} \tau+\mu_{3}^{\prime}+\mu_{3} \frac{\kappa^{\prime}}{\kappa}\right) B\right) \\
& b=\left(\mu_{3} \mu_{5}-\mu_{2} \mu_{6}\right) T+\left(\mu_{3} \mu_{4}-\mu_{1} \mu_{6}\right) N+\left(\mu_{1} \mu_{5}-\mu_{2} \mu_{4}\right) B
\end{aligned}
$$

where

$$
\begin{aligned}
& \mu_{1}=1+\kappa^{2} \sigma_{1}, \mu_{2}=\sigma_{1}^{\prime}+\sigma_{1} \frac{\kappa^{\prime}}{\kappa}-\sigma_{2} \tau, \mu_{3}=\sigma_{1} \tau+\sigma_{2}^{\prime}+\sigma_{2} \frac{\kappa^{\prime}}{\kappa}, \\
& \pi=\left(\left(1+\kappa^{2} \sigma_{1}\right)^{2}+\left(\sigma_{1}^{\prime}+\sigma_{1} \frac{\kappa^{\prime}}{\kappa}-\sigma_{2} \tau\right)^{2}+\left(\sigma_{1} \tau+\sigma_{2}^{\prime}+\sigma_{2} \frac{\kappa^{\prime}}{\kappa}\right)^{2}\right)^{1 / 2}, \\
& \mu_{4}=\frac{\mu_{1}^{\prime}+\mu_{2}^{\prime} \kappa^{2}}{\pi}, \mu_{5}=\frac{\mu_{1}+\mu_{2}^{\prime}+\mu_{2} \frac{\kappa^{\prime}}{\kappa}-\tau \mu_{3}}{\pi}, \mu_{6}=\frac{\mu_{2} \tau+\mu_{3}^{\prime}+\mu_{3} \frac{\kappa^{\prime}}{\kappa}}{\pi} .
\end{aligned}
$$

Theorem 3.9 Let $\alpha: I \rightarrow E_{1}^{3}$ be timelike curve with parametrized by arc-length in $\mathrm{E}_{1}^{3}$ and $P=\alpha+\sigma_{1} N+\sigma_{2} B$, where $\sigma_{1}$ is $\frac{1}{\kappa^{2}}, \sigma_{2}$ is $\frac{\sqrt{t^{2} \kappa^{2}-1}}{\kappa^{2}}$, be a parallel curve of $\alpha$. Then, adjoint curve of the parallel curve $P$ is

$$
\mathrm{A}=\int_{s_{0}}^{s}\left(\left(\mu_{3} \mu_{5}-\mu_{2} \mu_{6}\right) T+\left(\mu_{3} \mu_{4}-\mu_{1} \mu_{6}\right) N+\left(\mu_{1} \mu_{5}-\mu_{2} \mu_{4}\right) B\right)
$$

where

$$
\begin{aligned}
& \mu_{1}=1+\kappa^{2} \sigma_{1}, \mu_{2}=\sigma_{1}^{\prime}+\sigma_{1} \frac{\kappa^{\prime}}{\kappa}-\sigma_{2} \tau, \mu_{3}=\sigma_{1} \tau+\sigma_{2}^{\prime}+\sigma_{2} \frac{\kappa^{\prime}}{\kappa}, \\
& \pi=\left(\left(1+\kappa^{2} \sigma_{1}\right)^{2}+\left(\sigma_{1}^{\prime}+\sigma_{1} \frac{\kappa^{\prime}}{\kappa}-\sigma_{2} \tau\right)^{2}+\left(\sigma_{1} \tau+\sigma_{2}^{\prime}+\sigma_{2} \frac{\kappa^{\prime}}{\kappa}\right)^{2}\right)^{1 / 2}, \\
& \mu_{4}=\frac{\mu_{1}^{\prime}+\mu_{2}^{\prime} \kappa^{2}}{\pi}, \mu_{5}=\frac{\mu_{1}+\mu_{2}^{\prime}+\mu_{2} \frac{\kappa^{\prime}}{\kappa}-\tau \mu_{3}}{\pi}, \mu_{6}=\frac{\mu_{2} \tau+\mu_{3}^{\prime}+\mu_{3} \frac{\kappa^{\prime}}{\kappa}}{\pi} .
\end{aligned}
$$




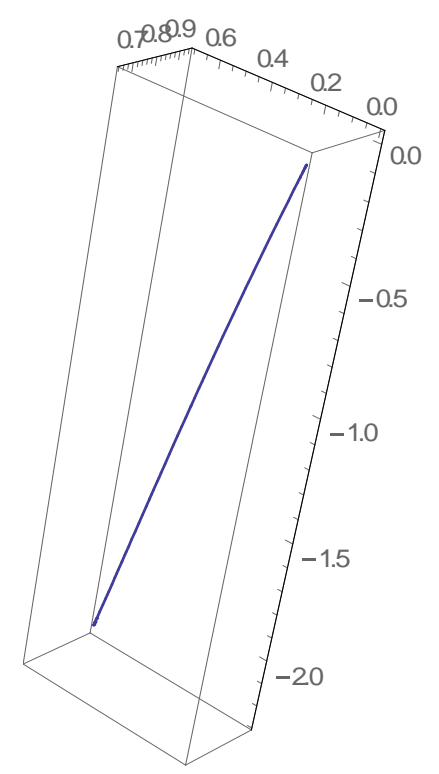

Fig. 3. The adjoint curve of $P$.

\section{Acknowledgment}

This work is supported by Selçuk University Scientic Research Projects Coordination Unit.

\section{References}

[1] Bukcu, B., \& Karacan, M. K. (2016). On the modified orthogonal frame with curvature and torsion in 3-space. Mathematical Sciences and Applications E-Notes, 4(1), 184-188.

[2] Bukcu, B., \& Karacan, M. K. (2016). Spherical curves with modified orthogonal frame. J. New Res. Sci., 10, 60-68.

[3] Körpınar, T., Turhan, E., \& Asil, V. (2011). Tangent bishop spherical images of a biharmonic b-slant helix in the heisenberg group heis3. Iranian Journal of Science and Technology Transaction A: Science A, 4(35), 265-271.

[4] Yllmaz, S., \& Turgut, M. (2010). A new version of bishop frame and an application to spherical images. J. Math. Anal. Appl., 371, 764-776.

[5] O'Neill, B. (1983). Semi Riemannian Geometry. New York: Academic Press.

[6] Yaglom, I. M., \& Shenitzer, A. (1979). A Simple Non-Euclidean Geometry and Its Pysical Basis. New York, Springer-Verlag.

[7] Chrastinova, V. (2007). Parallel curves in three-dimensional space. Sbornik 5. Konference o Matematice a Fyzice, UNOB.

[8] Nurkan, S. K., Güven, İ. A., \& Karacan, M. K. (2018). Characterizations of adjoint curves in euclidean 3-space. Proc. Natl. Acad. Sci., India, Sect. A Phys. Sci.

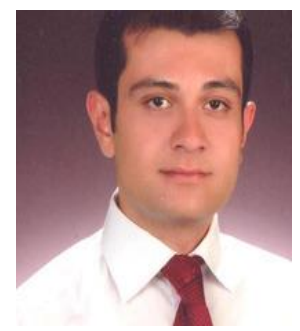

Muhammed Talat Sariaydın is an assistant professor in the Department of Mathematics, Selçuk University, Konya, Turkey. He received his B.Sc., M.Sc., and PhD in mathematics from Firat University of Turkey. His research interests include geometric modeling, and geometry of curves and surfaces in Riemannian and semi-Riemannian geometries. 


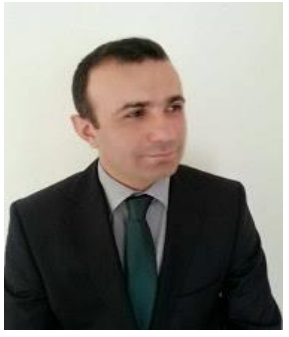

Talat Körpınar is an associate professor in the Department of Mathematics, Muş Alparslan University, Muş, Turkey. He received his B.Sc., M.Sc., and PhD in mathematics from Cumhuriyet University and Firat University of Turkey, respectively. His research interests include geometry of curves and surfaces in Euclidean and Lorentzian geometries. 